\title{
Anticholinergic drug use and its association with self-reported symptoms among older persons with and without diabetes
}

\author{
Inkeri, Niina-Mari
}

2019-04

Inkeri , N-M , Karjalainen , M , Haanpaa , M , Kautiainen , H , Saltevo , J , Mantyselka , P \&

Tiihonen, M 2019 , ' Anticholinergic drug use and its association with self-reported symptoms among older persons with and without diabetes ' , Journal of Clinical Pharmacy and Therapeutics, vol. 44 , no. 2 , pp. 229-235 . https://doi.org/10.1111/jcpt.12772

http://hdl.handle.net/10138/313506

https://doi.org/10.1111/jcpt.12772

unspecified

publishedVersion

Downloaded from Helda, University of Helsinki institutional repository.

This is an electronic reprint of the original article.

This reprint may differ from the original in pagination and typographic detail.

Please cite the original version. 


\title{
Anticholinergic drug use and its association with self-reported symptoms among older persons with and without diabetes
}

\author{
Niina-Mari Inkeri MSc (Pharm) $)^{1}$ ～Merja Karjalainen MD $^{2,3} \mid$ Maija Haanpää MD, $\mathrm{PhD}^{4,5}$ | \\ Hannu Kautiainen $\mathrm{BA}^{6,7}$ ） Juha Saltevo MD, $\mathrm{PhD}^{8}$ | Pekka Mäntyselkä MD, $\mathrm{PhD}^{2,7}$ | \\ Miia Tiihonen PhD (Pharm) ${ }^{1}$
}

\footnotetext{
${ }^{1}$ School of Pharmacy, University of Eastern Finland, Kuopio, Finland

${ }^{2}$ Institute of Public Health and Clinical Nutrition, General Practice, University of Eastern Finland, Kuopio, Finland

${ }^{3}$ Inner Savo Health Center, Suonenjoki,

Finland

${ }^{4}$ IImarinen Mutual Pension Insurance Company, Vantaa, Finland

${ }^{5}$ Department of Neurosurgery, Helsinki University Hospital, Helsinki, Finland

${ }^{6}$ Unit of Primary Health Care, Helsinki University Central Hospital, Helsinki, Finland

${ }^{7}$ Primary Health Care Unit, Kuopio University Hospital, Kuopio, Finland

${ }^{8}$ Central Finland Central Hospital, Jyväskylä, Finland
}

\section{Correspondence}

Miia Tiihonen, School of Pharmacy, University of Eastern Finland, Kuopio, Finland.

Email: miia.tiihonen@uef.fi

Funding information

$\mathrm{N}-\mathrm{M}$.I has received a personal grant from the Urho and Kaisu Kiukas Foundation and M.K has received a personal grant from the North Savo Cultural Foundation.

\section{Summary}

What is known and objective: Anticholinergic drug use has been associated with a risk of central and peripheral adverse effects. There is a lack of information on anticholinergic drug use in persons with diabetes. The aim of this study is to investigate anticholinergic drug use and the association between anticholinergic drug use and self-reported symptoms in older community-dwelling persons with and without diabetes.

Methods: The basic population was comprised of Finnish community-dwelling primary care patients aged 65 and older. Persons with diabetes were identified according to the ICD-10 diagnostic codes from electronic patient records. Two controls adjusted by age and gender were selected for each person with diabetes. This crosssectional study was based on electronic primary care patient records and a structured health questionnaire. The health questionnaire was returned by $430(81.6 \%)$ persons with diabetes and 654 (73.5\%) persons without diabetes. Data on prescribed drugs were obtained from the electronic patient records. Anticholinergic drug use was measured according to the Anticholinergic Risk Scale. The presence and strength of anticholinergic symptoms were asked in the health questionnaire.

Results and discussion: The prevalence of anticholinergic drug use was $8.9 \%$ in the total study cohort. There were no significant differences in anticholinergic drug use between persons with and without diabetes. There was no consistent association between anticholinergic drug use and self-reported symptoms.

What is new and conclusion: There is no difference in anticholinergic drug use in older community-dwelling persons with and without diabetes. Anticholinergic drug use should be considered individually and monitored carefully.

\section{KEYWORDS}

anticholinergic burden, anticholinergic drug use, diabetes mellitus, older people

\section{1 | WHAT IS KNOWN AND OBJECTIVE}

Several drugs prescribed for common indications have anticholinergic properties. ${ }^{1}$ Anticholinergic properties may be desired in the management of certain conditions (eg, urinary incontinence and Parkinson's disease). Numerous common drugs in different therapeutic groups may have anticholinergic properties, and identifying these properties may be challenging. Diabetes has been associated 
with conditions for which drugs with anticholinergic properties may be prescribed (eg, depression and neuropathic pain). ${ }^{2,3}$

Anticholinergic drugs expose older people to adverse drug effects. ${ }^{4}$ Older people are known to be particularly at increased risk of anticholinergic adverse effects due to a decrease in central cholinergic receptors and an increase in blood-brain barrier permeability. ${ }^{5,6}$ Anticholinergic adverse drug effects may occur peripherally (eg, dry mouth, dry eyes and constipation) or centrally (eg, drowsiness, dizziness and confusion). ${ }^{4}$ Furthermore, anticholinergic drug use has been associated with both cognitive and functional decline. ${ }^{7-9}$ Anticholinergic drug use in older people may lead to further adverse health outcomes, including hospital admissions and more visits to general practitioners. ${ }^{10}$

There is limited previous research on anticholinergic drug use in specific populations. Anticholinergic drug use has been evaluated, for example in persons with cognitive impairment, ${ }^{7}$ schizophrenia ${ }^{11}$ and Parkinson's disease. ${ }^{12,13}$ There are a few previous studies investigating anticholinergic drug use in persons with diabetes. ${ }^{14,15}$ These studies have focused on management of overactive bladder syndrome. Anticholinergic drug use has been associated with a higher prevalence of diabetes in a previous Finnish study. ${ }^{16}$

Diabetes has been associated with polypharmacy and comorbidities. ${ }^{17,18}$ In particular, people with type 2 diabetes are at a greater risk of polypharmacy. ${ }^{19}$ Polypharmacy in people with diabetes can be justifiable. Nevertheless, polypharmacy has been associated with anticholinergic drug use and a greater anticholinergic burden. ${ }^{20-23}$ Furthermore, diabetes has been associated with possible bloodbrain barrier dysfunction, which may increase the risk of central adverse effects. ${ }^{24-26}$

The aim of this study was to evaluate anticholinergic drug use and its association with self-reported symptoms among communitydwelling older people with and without diabetes.

\section{2 | METHODS}

\section{1 | Study population}

This cross-sectional study was conducted in the communities of Suonenjoki and Rautalampi in Eastern Finland. The total population of the district was 10793 , and the basic population $(\mathrm{N}=3093)$ was comprised of community-dwelling primary care patients aged 65 and older. Persons with diabetes $(N=540)$ were identified from primary care electronic patient records according to diagnostic codes E10 and E11 of the International Classification of Diagnoses (ICD-10). ${ }^{27}$ The cohort included 12 subjects with type 1 diabetes. Two controls adjusted by age and gender were selected for each person with diabetes. A structured health questionnaire was mailed once to 527 persons with diabetes and 890 controls in 2015 (August-September). This study is a part of the ISDM study, which has been described previously elsewhere by Karjalainen et al. ${ }^{28}$

\section{2 | Study design}

\subsection{1 | Data source}

Data on prescribed drugs were obtained from the primary care electronic patient records (Pegasos ${ }^{\circledR}$, CGI Suomi Ltd, Helsinki, Finland). Regular medications (daily or at regular intervals) were considered. Medications were categorized according to the World Health Organization (WHO) Anatomical Therapeutic Chemical (ATC) classification system codes. ${ }^{29}$

\subsubsection{Anticholinergic burden}

The Anticholinergic Risk Scale (ARS) created by Rudolph et al is one of several tools available for identifying a cumulative anticholinergic burden that may lead to adverse effects. ${ }^{4,30}$ In this study, the anticholinergic burden was quantified using the ARS, which has been previously applied in numerous studies. ${ }^{30}$ The ARS is based on a literature review and expert opinion. ${ }^{4}$ The review encompassed the 500 most frequently prescribed medications within the Veterans Affairs Boston Healthcare System, excluding topical, ophthalmic, otologic and inhaled medication preparations. Medications were classified into four categories from 0 to 3 according to their anticholinergic activity ( 0 , limited or none; 1 , moderate; 2 , strong; 3 , very strong). At present, 40 of the 49 anticholinergic medications listed in the ARS are available in Finland. ${ }^{31}$

\subsection{3 | Measurements and tools}

A structured health questionnaire was used to obtain data on background variables (sex, age, education years, living arrangements), self-rated health, health-related quality of life, exercise, depressive symptoms, consumption of alcohol, smoking, comorbidities and selfreported symptoms.

Self-rated health was assessed based on a numerical rating scale from 1 to 5 (excellent-poor). Health-related quality of life was assessed using the EuroQol EQ-5D questionnaire. ${ }^{32}$ The frequency, efficiency and duration of exercise were assessed based on Kasari's FIT Index. ${ }^{33}$ Depressive symptoms were evaluated using the Geriatric Depression Scale (GDS-15). ${ }^{34}$ Alcohol consumption was measured based on the Alcohol Use Disorders Identification Test (AUDIT-C). ${ }^{35}$ History and frequency of smoking were asked. Comorbidity was defined according to a list of common chronic diseases (mental disorder, Parkinson's disease, dementia, musculoskeletal disorder, cardiovascular disease, cancer, chronic obstructive pulmonary disease, asthma) and calculated using the Charlson Comorbidity Index $(\mathrm{CCl}){ }^{36}$

In one part of the questionnaire, the participants were asked to assess whether they had experienced various symptoms: dry eyes, dry mouth, constipation, gastrointestinal dysfunction or dizziness. These symptoms present typical anticholinergic adverse effects in older people. ${ }^{4}$ The participants ranked the severity of each symptom using a numerical rating scale from 0 to 10 . 


\section{3 | Ethics approval}

The study was approved by the Inner Savo Health Care Federation of Municipalities (61 A/2015). The study protocol of the ISDM (Inner Savo Diabetes Mellitus) study was approved by the Research Ethics Committee of the Northern Savo Hospital District, Kuopio, Finland (256/2015). The study was conducted in accordance with the Helsinki's Declaration. The health questionnaire included an information letter explaining the use of the data, and returning the questionnaire was voluntary. The autonomy of the research subjects was respected, and only anonymous data were analysed. No harm to the subjects was possible, and the confidentiality of the subjects and research data was protected.

\section{4 | Statistical analysis}

Descriptive statistics include means and SDs (standard deviations) for continuous variables and numbers and percentages for categorical variables. Statistical comparisons were made using analysis of variance (ANOVA) and logistic models with covariates when appropriate. Models included groups (diabetes and the ARS) and their interaction. The STATA 15.0, StataCorp LP (College Station, TX, USA) statistical package, was used for the analyses.

\section{3 | RESULTS AND DISCUSSION}

The health questionnaire was returned by 430 persons with diabetes (81.6\%) and 654 controls (73.5\%). Females constituted $50.9 \%$ of the respondents with diabetes and $54.4 \%$ of the respondents without diabetes. The demographic characteristics of the study cohort according to anticholinergic drug use are presented in Table 1.

Persons with diabetes had a higher mean age, fewer years of education, a lower EQ-5D score, a lower Kasari's FIT Index, mobility aids more often, a higher $\mathrm{CCl}$ (excluding diabetes), dementia more often, cardiovascular disease more often and a higher mean number of drugs compared with persons without diabetes. Persons using at least one anticholinergic drug were more often female, had a higher mean age, were more often

TABLE 1 Descriptive characteristics of the study population

\begin{tabular}{|c|c|c|c|c|c|c|c|}
\hline & \multicolumn{2}{|c|}{ No diabetes } & \multicolumn{2}{|l|}{ Diabetes } & \multicolumn{3}{|c|}{$P$-values for effects } \\
\hline Age, mean (SD) & $74(6)$ & $75(7)$ & $75(7)$ & $77(8)$ & 0.024 & 0.009 & 0.23 \\
\hline Education, y, mean (SD) & $9.9(3.2)$ & $9.9(3.3)$ & $9.3(3.0)$ & $9.2(3.0)$ & 0.006 & 0.89 & 0.64 \\
\hline Good self-rated health $(1,2,3), \mathrm{n}(\%)$ & $343(57.3)$ & $21(38.2)$ & $165(42.4)$ & $13(31.7)$ & 0.052 & 0.007 & 0.49 \\
\hline EQ-5D, mean (SD) & $0.8(0.2)$ & $0.7(0.2)$ & $0.7(0.2)$ & $0.6(0.3)$ & 0.004 & $<0.001$ & 0.80 \\
\hline No mobility aids, n (\%) & $502(83.8)$ & $45(81.8)$ & $279(71.7)$ & $20(48.8)$ & $<0.001$ & 0.024 & 0.090 \\
\hline Kasari's FIT Index, mean (SD) & $40(22)$ & $37(24)$ & $30(22)$ & $24(16)$ & $<0.001$ & 0.084 & 0.51 \\
\hline \multicolumn{8}{|l|}{$\mathrm{CCl}$, mean (SD) } \\
\hline Total & $0.4(0.7)$ & $0.5(0.7)$ & $1.7(1.0)$ & $1.9(1.1)$ & $<0.001$ & 0.051 & 0.44 \\
\hline Excluding diabetes & $0.4(0.7)$ & $0.5(0.7)$ & $0.6(0.9)$ & $0.7(1.0)$ & 0.011 & 0.14 & 0.65 \\
\hline Mental disorder, n (\%) & $8(1.3)$ & $4(7.3)$ & $14(3.6)$ & $4(9.8)$ & 0.12 & 0.001 & 0.42 \\
\hline Parkinson's disease, n (\%) & $2(0.3)$ & $3(5.5)$ & $5(1.3)$ & $2(4.9)$ & 0.32 & $<0.001$ & 0.24 \\
\hline Dementia, n (\%) & $27(4.5)$ & $3(5.5)$ & $27(6.9)$ & 7 (17.1) & 0.026 & 0.12 & 0.29 \\
\hline Musculoskeletal disorder, $\mathrm{n}(\%)$ & $257(42.9)$ & $34(61.8)$ & $206(53.0)$ & $29(70.7)$ & 0.081 & $<0.001$ & 0.99 \\
\hline Cardiovascular disease, n (\%) & $337(56.3)$ & $36(65.5)$ & $302(77.6)$ & $34(82.9)$ & $<0.001$ & 0.17 & 0.92 \\
\hline Cancer C00-97 (malign tumour), n (\%) & $52(8.7)$ & $1(1.8)$ & $26(6.7)$ & $2(4.9)$ & 0.32 & 0.25 & 0.54 \\
\hline
\end{tabular}

ATC codes: P, antiparasitic products, insecticides and repellents; D, dermatologicals; J, anti-infectives for systemic use; V, various (eg, allergens, diagnostic agents) ${ }^{27}$; ARS, Anticholinergic Risk Scale ${ }^{2}$; AUDIT-C, alcohol use disorders identification test ${ }^{33}$; $\mathrm{CCI}$, Charlson Comorbidity Index ${ }^{34}$; COPD, chronic obstructive pulmonary disease; EQ-5D; EuroQol EQ-5D questionnaire ${ }^{30}$; GDS-15, Geriatric Depression Scale. ${ }^{32}$ 
living alone, less frequently had good self-rated health, had a lower EQ-5D score, more often had mobility aids, had higher mean GDS-15 scores, more often had a mental disorder, Parkinson's disease, a musculoskeletal disorder, COPD or asthma and had a higher mean number of drugs. There was no interaction between the groups (diabetes and the ARS).

\section{1 | Anticholinergic drug use}

The use of anticholinergic drugs is described in detail in Table 2. The prevalence of anticholinergic drug use was $8.9 \%$ in the total study cohort. Anticholinergic drug use was not associated with having diabetes. Forty-one (9.5\%) persons with diabetes had at least one ARS score, whereas 55 (8.4\%) of the controls scored at least one. The highest level of anticholinergic burden was five scores (four of the persons without diabetes).

The anticholinergic drugs used in the cohort are shown in Table 3. The most frequently used anticholinergic drug groups were psychoanaleptics (32.1\% of total anticholinergic use), antihistamines for systemic use (20.8\%) and psycholeptics (19.8\%) among both persons with and without diabetes. Twenty-four anticholinergic drugs were identified. The most frequently used anticholinergic drugs in the total cohort were mirtazapine (12.3\%), cetirizine (12.3\%), amitriptyline with psycholeptics (10.4\%), amitriptyline (8.5\%) and loratadine (8.5\%).

\subsection{Anticholinergic drug use and self- reported symptoms}

There was no consistent association between anticholinergic drug use and self-reported symptoms. Anticholinergic drug use was associated with a higher risk of constipation and dizziness in both groups when adjusted by age, gender, years of education, GDS-15, smoking, alcohol consumption and $\mathrm{CCl}$ (excluding diabetes). In addition to this, anticholinergic drug use was associated with a higher risk of dry eyes in the group with diabetes and a higher risk of dry mouth, gastrointestinal dysfunction and fatigue in the control group (in the adjusted model). The results in the adjusted model are shown in Figure 1.

\section{4 | DISCUSSION}

One-tenth of this older community-dwelling Finnish population used anticholinergic drugs, and anticholinergic drug use was not associated with having diabetes. The results suggest that the use of anticholinergic drugs in the study population is relatively low. In previous Finnish studies, the prevalence of anticholinergic drug use has varied from $16 \%$ among community dwellers to $55 \%$ among persons living in long-term wards (when measured using the ARS). ${ }^{20,37,38}$ There are no known previous studies investigating the prevalence of anticholinergic drug use in persons with diabetes, whereupon it is not possible to compare the results with previous findings in similar populations. Persons with diabetes may be even better monitored because the healthcare system provides diabetes nurse services, for example. However, one previous Finnish study found diabetes to be more common in anticholinergic drug users than in non-users among home-dwelling older people, which shows that the association is unclear. ${ }^{16}$ The disparity of the results may arise from differences between the study populations and anticholinergic drug scales.

Psychoanaleptics and psycholeptics composed approximately half of the anticholinergic drug use in the study cohort. These findings are mostly in line with a previous Finnish study using the ARS, which found risperidone, mirtazapine, olanzapine and hydroxyzine as the most frequently used anticholinergic drugs among persons living in long-term wards. ${ }^{37}$ Low-dose administration of mirtazapine in the management of chronic insomnia is mentioned in the Finnish Good Care Guidelines. ${ }^{39}$ Mirtazapine might have been chosen as a safer alternative to benzodiazepines or $z$-drugs.

In this study, we investigated the association between anticholinergic drug use and self-reported symptoms that may indicate adverse drug effects. There was no consistent association between anticholinergic drug use and symptoms evaluated although the ARS, and the association with anticholinergic symptoms has been validated. ${ }^{4}$ Similarly with our study, a previous Finnish study did not find a consistent association between anticholinergic drug use and adverse drug events. ${ }^{20}$ Anticholinergic drug use was defined according to the ARS, but the adverse outcomes differed from those in our study (eg, functional capacity and cognition). Another Finnish study using the ARS did not find an association between anticholinergic drug use and dry mouth or constipation. ${ }^{38}$ Studies using different anticholinergic drug scales have shown dry mouth and constipation to be more prevalent among anticholinergic drug users. ${ }^{23,40} \mathrm{We}$ were not able to confirm the cause of symptoms; the symptoms may be both drug-related and disease-related.

One of the strengths of this study was the high response rate of the health questionnaire and the high validity of the data collection.

\begin{tabular}{lccc}
$\begin{array}{l}\text { Rudolph's Anticholinergic Risk } \\
\text { Scale (score) }\end{array}$ & No diabetes, $\mathbf{n}(\%)$ & Diabetes, $\mathbf{n}(\%)$ & Total, $\mathbf{n}(\%)$ \\
\hline 0 & $599(91.6)$ & $389(90.5)$ & $988(91.1)$ \\
\hline 1 & $18(2.8)$ & $12(2.8)$ & $30(2.8)$ \\
\hline 2 & $14(2.1)$ & $15(3.5)$ & $29(2.7)$ \\
\hline 3 & $19(2.9)$ & $12(2.8)$ & $31(2.9)$ \\
\hline 4 & $0(0.0)$ & $2(0.5)$ & $2(0.2)$ \\
\hline 5 & $4(0.6)$ & $0(0.0)$ & $4(0.4)$ \\
\hline
\end{tabular}

TAB LE 2 Anticholinergic drug use among persons with and without diabetes 
TAB LE 3 Anticholinergic drugs used by persons with and without diabetes

\begin{tabular}{|c|c|c|c|}
\hline Anticholinergic drug (ATC code) & No diabetes, $\mathrm{n}(\%)$ & Diabetes, $\mathrm{n}(\%)$ & Total, n (\%) \\
\hline Mirtazapine (N06AX11) & $8(12.9)$ & $5(11.4)$ & $13(12.3)$ \\
\hline Amitriptyline (N06AA09) & $5(8.1)$ & $4(9.1)$ & $9(8.5)$ \\
\hline Loratadine (R06AX13) & $7(11.3)$ & $2(4.6)$ & $9(8.5)$ \\
\hline Quetiapine (N05AH04) & $1(1.6)$ & $5(11.4)$ & $6(5.7)$ \\
\hline Hydroxyzine (N05BB01) & $1(1.6)$ & $3(6.8)$ & $4(3.8)$ \\
\hline Levodopa and decarboxylase inhibitor (N04BA02) & $3(4.8)$ & $1(2.3)$ & $4(3.8)$ \\
\hline Olanzapine (N05AH03) & $2(3.2)$ & $2(4.6)$ & $4(3.8)$ \\
\hline Pseudoephedrine, combinations (R01BA52) & $2(3.2)$ & $1(2.3)$ & $3(2.8)$ \\
\hline Metoclopramide (A03FA01) & $0(0.0)$ & $2(4.6)$ & $2(1.9)$ \\
\hline Oxybutynin (G04BD04) & $1(1.6)$ & $1(2.3)$ & $2(1.9)$ \\
\hline Baclofen (M03BX01) & $0(0.0)$ & $1(2.3)$ & $1(0.9)$ \\
\hline Clozapine (N05AH02) & $0(0.0)$ & $1(2.3)$ & $1(0.9)$ \\
\hline Levodopa, decarboxylase inhibitor and COMT inhibitor (N04BA03) & $0(0.0)$ & $1(2.3)$ & $1(0.9)$ \\
\hline Perphenazine (N05AB03) & $1(1.6)$ & $0(0.0)$ & $1(0.9)$ \\
\hline Prochlorperazine (N05AB04) & $1(1.6)$ & $0(0.0)$ & $1(0.9)$ \\
\hline Ranitidine (A02BA02) & $1(1.6)$ & $0(0.0)$ & $1(0.9)$ \\
\hline
\end{tabular}

ATC, anatomical therapeutic chemical.

FIGURE 1 Self-reported symptoms among persons with and without diabetes (adjusted model). ARS, Anticholinergic Risk Scale; NRS, numerical rating scale

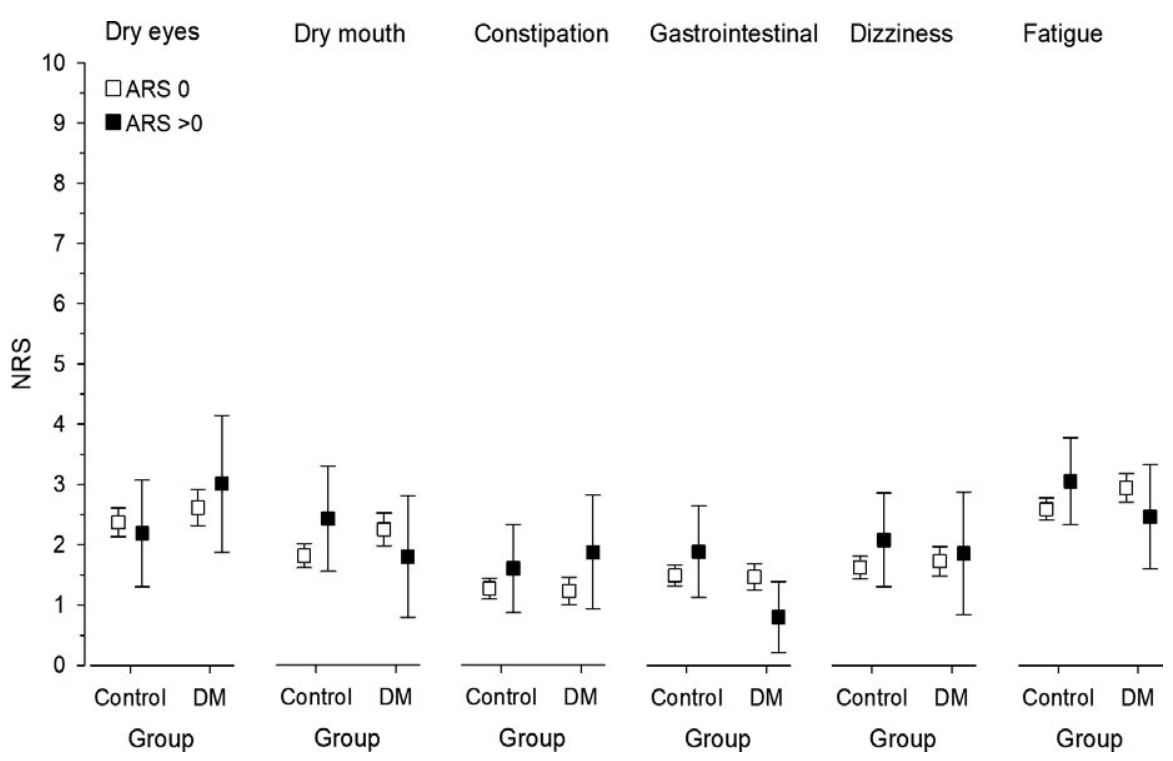

The health questionnaire provided thorough information on background variables, which enables comparison of the groups and adjustment for several potential confounders. The ARS has been validated in a veteran's population, and higher ARS scores have been associated with anticholinergic adverse effects. ${ }^{4}$ Apart from that, the ARS has been previously applied to other studies evaluating the 
association between anticholinergic medications and adverse drug effects. ${ }^{20,38}$ Additionally, not only the presence but also the strength of symptoms was determined in this study.

This study has a few limitations. The study cohort was geographically restricted and represents the population of one primary care district in a semirural area in Eastern Finland. The findings cannot be directly generalized to other populations. Persons with diabetes represent persons able to live at home, which makes survival bias possible. Because of the cross-sectional design of the study, it was not possible to prove any causal relationship between anticholinergic drug use and self-reported symptoms. Although we obtained extensive information on background variables, only diagnosis of diabetes was confirmed from electronic patient records; other comorbidities were self-reported. We were not able to determine the onset or progression of diabetes exactly, but we can presume that the participants represent patients with a substantially long history of diabetes, since the study recruitment was performed according to an established diagnosis of diabetes 3 months before the questionnaire was sent. Lastly, self-reporting may underestimate adverse effects compared with adverse effects noted in the clinical examination. $^{41}$

\section{WHAT IS NEW AND CONCLUSION}

There is no difference in anticholinergic drug use in older community-dwelling persons with and without diabetes. There is no consistent association between anticholinergic drug use and self-reported anticholinergic symptoms. Further studies are needed to confirm the impact of anticholinergic drugs on experienced symptoms in different subpopulations.

\section{INFORMED CONSENT}

The health questionnaire included an information letter explaining the use of the data, and returning the questionnaire was voluntary. The autonomy of the research subjects was respected, and only anonymous data were analysed. No harm to the subjects was possible, and the confidentiality of the subjects and research data was protected.

\section{ETHICAL APPROVAL}

All procedures performed in studies involving human participants were in accordance with the ethical standards of the Research Ethics Committee of the Northern Savo Hospital District, Kuopio, Finland (256/2015), and with the 1964 Helsinki's declaration and its later amendments or comparable ethical standards.

\section{CONFLICT OF INTEREST}

No conflicts of interest have been declared.
ORCID

Miia Tiihonen (iD http://orcid.org/0000-0002-5069-5045

\section{REFERENCES}

1. The American Geriatrics Society Beers Criteria Update Expert Panel. American Geriatrics Society updated Beers Criteria for potentially inappropriate medication use in older adults. J Am Geriatr Soc. 2015;2015(63):2227-2246.

2. Anderson RJ, Freedland KE, Clouse RE, Lustman PJ. The prevalence of comorbid depression in adults with diabetes. Diabetes Care. 2001;24:1069-1078.

3. Peltier A, Goutman SA, Callaghan BC. Painful diabetic neuropathy. BMJ. 2014;348:g1799.

4. Rudolph JL, Salow MJ, Angelini MC, McGlinchey R. The anticholinergic risk scale and anticholinergic adverse effects in older persons. Arch Intern Med. 2008;168:508-513.

5. Pakulski C, Drobnik L, Millo B. Age and sex as factors modifying the function of the blood-cerebrospinal fluid barrier. Med Sci Monit. 2000;6:314-318.

6. Lonsdale $D$, Baker E. Understanding and managing medication in elderly people. Best Pract Res Clin Obstet Gynaecol. 2013;27:767-788.

7. Fox C, Richardson K, Maidment ID, et al. Anticholinergic medication use and cognitive impairment in the older population: the Medical Research Council Cognitive Function and Ageing Study. J Am Geriatr Soc. 2011;59:1477-1483.

8. Pasina L, Djade C, Lucca U, et al. Association of anticholinergic burden with cognitive and functional status in a cohort of hospitalized elderly: comparison of the Anticholinergic Cognitive Burden Scale and Anticholinergic Risk Scale. Results from the REPOSI study. Drugs Aging. 2013;30:103-112.

9. Fox C, Smith T, Maidment ID. Effect of medications with anti-cholinergic properties on cognitive function, delirium, physical function and mortality: a systematic review. Drugs Aging. 2014;43:604-615.

10. Salahudeen M, Hilmer S, Nishtala P. Comparison of anticholinergic risk scales and associations with adverse health outcomes in older people. J Am Geriatr Soc. 2015;63:85-90.

11. Pristed SG, Correll CU, Nielsen J. Frequency and correlates of anticholinergic use among patients with schizophrenia in Denmark: a nation-wide pharmacoepidemiological study. Psychiatry Res. 2017;225:198-203.

12. Ehrt U, Broich K, Larsen J, Ballard C, Aarsland D. Use of drugs with anticholinergic effect and impact on cognition in Parkinson's disease: a cohort study. J Neurol Neurosurg Psychiatry. 2010;81:160-165.

13. Crispo J, Willis A, Thibault $D$, et al. Associations between anticholinergic burden and adverse health outcomes in Parkinson disease. PLoS One. 2016;11(3):e0150621.

14. Schneider T, Marschall-Kehrel D, Hanisch JU, Michel MC. Does concomitant diabetes affect treatment responses in overactive bladder patients? Int J Clin Pract. 2013;67:1138-1143.

15. Yang YW, Liu HH, Chuang HY, Hsieh T. Association between different anticholinergic drugs and subsequent dementia risk in patients with diabetes mellitus. PLoS One. 2017;12(4):e0175335.

16. Uusvaara J, Pitkälä KH, Kautiainen H, Tilvis RS, Strandberg TE. Association of anticholinergic drugs with hospitalization and mortality among older cardiovascular patients: a prospective study. Drugs Aging. 2001;28:131-138.

17. Formiga $F$, Vidal $X$, Agusti $A$, et al. Inappropriate prescribing in elderly people with diabetes admitted to hospital. Diabet Med. 2016;18(33):655-662.

18. Andreassen L, Kjome R, Solvik U, Houghton J, Desborough J. The potential for deprescribing in care home residents with type 2 diabetes. Int J Clin Pharm. 2016;30(38):977-984. 
19. Bauer S, Nauck M. Polypharmacy in people with type 1 and type 2 diabetes is justified by current guidelines - a comprehensive assessment of drug prescriptions in patients needing inpatient treatment for diabetes-associated problems. Diabet Med. 2014;31:1078-1085.

20. Lampela P, Lavikainen P, Garcia-Horsman JA, Bell JS, Hartikainen S. Anticholinergic drug use, serum anticholinergic activity, and adverse drug events among older people: a population-based study. Drugs Aging. 2013;30:321-330.

21. Mate KE, Kerr KP, Pond D, et al. Impact of multiple low-level anticholinergic medications on anticholinergic load of community-dwelling elderly with and without dementia. Drugs Aging. 2015;32:159-167.

22. Gutiérrez-Valencia $M$, Martinéz-Velilla $N$, Vetrano DL. Anticholinergic burden and health outcomes among older adults discharged from hospital: results from the CRIME study. Eur J Clin Pharmacol. 2017;73:1467-1474.

23. Tiisanoja A, Syrjälä AM, Komulainen K, et al. Anticholinergic burden and dry mouth among Finnish, community dwelling older adults. Gerodontology. 2018;35(1):3-10.

24. Starr J, Wardlaw J, Ferguson K, MacLullich A, Deary I, Marshall I. Increased blood-brain permeability in type II diabetes demonstrated by gadolinium magnetic resonance imaging. I Neurol Neurosurg Psychiatry. 2003;74:70-76.

25. Serlin Y, Levy J, Shlaev H. Vascular pathology and blood-brain barrier disruption in cognitive and psychiatric complications of type 2 diabetes mellitus. Cardiovasc Psychiatry Neurol. 2011;2011:1-10.

26. Janelidze $S$, Hertze J, Nägga $K$, et al. Increased blood-brain barrier permeability is associated with dementia and diabetes but not amyloid pathology or APOE genotype. Neurobiol Aging. 2017;51:104-112.

27. WHO. The international classification of diseases (ICD-10) diagnostic codes, 2017. https://www.who.int/classifications/icd/en/. Accessed April 10, 2017.

28. Karjalainen M, Tiihonen $M$, Kautiainen $H$, Saltevo J, Haanpää $M$, Mäntyselkä P. Pain and self-rated health in older people with and without type 2 diabetes. Eur Geriatr Med. 2018;9:127-131.

29. $\mathrm{WHO}$ (WHO Collaborating Centre for Drug Statistics Methodology). International language for drug utilization research. https://www. whocc.no. Accessed May 9, 2017.

30. Salahudeen MS, Duffull SB, Nishtala PS. Anticholinergic burden quantified by anticholinergic risk scales and adverse outcomes in older people: a systematic review. BMC Geriatr. 2015;15(1):31.

31. Duodecim. Medicine Database. Helsinki, Finland: Kustannus Oy Duodecim. Publishing company Duodecim; 2018. (Finnish).
32. EuroQol Group. EuroQol - a new faculty for the measurement of health-related quality of life. Health Policy. 1990;163:199-208.

33. Hicks VL, Solarcyzyk LM, Heyward VH, Baumgartner RN. Validation on near-infrared interactance and skinfold methods for estimating body composition of American Indian women. Med Sci Sports Exerc. 2000;32:531-539.

34. Sheik JY, Sheik J, Yesavage J. Geriatric Depression Scale (GDS): recent evidence and development of a shorter version. Clin Gerontol. 1986;5:165-172.

35. Saunders JB, Aasland OG, Babor TF, De laFuente JR, Grant M. Alcohol Use Disorders Identification Test (AUDIT): WHO collaborative project on early detection of persons with harmful alcohol consumption. Addiction. 1993;88:791-804.

36. Charlson ME, Pompei P, Ales KL, MacKenzie CR. A new method of classifying prognostic comorbidity in longitudinal studies: development and validation. J Chronic Dis. 1987;40:373-383.

37. Kumpula EK, Bell J, Soini H, Pitkälä KH. Anticholinergic drug use and mortality among residents of long-term care facilities: a prospective cohort study. J Clin Pharmacol. 2011;51:256-263.

38. Aalto AL, Roitto HM, Finne-Soveri H, Kautiainen H, Pitkälä K. Use of anticholinergic drugs and its relationship with psychological wellbeing and mortality in long term facilities in Helsinki. J Am Med Dir Assoc. 2018;19:511-515.

39. Insomnia. Current Care Guidelines. Working Group Set up by the Finnish Medical Society Duodecim, the Finnish Sleep Research Society. Helsinki, Finland: The Finnish Medical Society Duodecim; 2017. (in Finnish).

40. Ness J, Hoth A, Barnett MJ, Shorr RI, Kaboli PJ. Anticholinergic medications in community-dwelling older veterans: prevalence of anticholinergic symptoms, symptom burden, and adverse drug events. Am J Geriatr Pharmacother. 2006;4:42-51.

41. Lampela P, Hartikainen S, Sulkava R, Huupponen R. Adverse drug effects in elderly people - a disparity between clinical examination and adverse effects self-reported by the patient. Eur J Clin Pharmacol. 2007;63:509-515.

How to cite this article: Inkeri N-M, Karjalainen M, Haanpää $M$, et al. Anticholinergic drug use and its association with selfreported symptoms among older persons with and without diabetes. J Clin Pharm Ther. 2019;44:229-235. https://doi. org/10.1111/jcpt.12772 